\title{
Análise do perfil epidemiológico dos pacientes acometidos por doença de chagas aguda notificados em Araguaína-TO no período de 2007 a 2018
}

\section{Analysis of the epidemiological profile of patients affected by acute chagas disease notified in Araguaína-TO in the period 2007 to 2018}

Diego Santos Andrade1, Brenda Pereira Teles², Daiene Isabel da Silva Lopes 3 , Durval Nolasco das Neves Neto 4.

\section{RESUMO}

Os conhecimentos sobre a doença de Chagas tiveram seu pontapé inicial no ano de 1909, através de uma expressiva endemia rural que ocorreu em vários países latinoamericanos. A doença de Chagas (DC) é uma patologia de cunho infeccioso, provocada pelo agente etiológico Trypanosoma cruzi sendo resultante das alterações humanas no meio ambiente. Com a doença em curso, ocorre uma agressão progressiva ao coração, caracterizada por uma miocardite, tem como resultado a cardiomiopatia crônica da doença de Chagas (CCDC). A fase aguda desta doença inicia-se logo depois do período de incubação, que ocorre de 1-4 semanas após a exposição ao T. cruzi, sendo o período em que não há manifestação clínica após a contaminação denominado de período de incubação. Como é uma doença infecciosa, o diagnóstico etiológico da doença de Chagas no Brasil tem que ser realizado em todos os casos suspeitos, na fase aguda e na fase crônica. Desse modo, é fundamental integrar evidências epidemiológicas, clínicas e laboratoriais, a fim de se aumentar o grau de predição e a acurácia do diagnóstico.

Palavras-chave: Doença de Chagas aguda. Trypanosoma cruzi. Barbeiro.

\section{ABSTRACT}

The knowledge about Chagas' disease started in 1909, through an expressive rural endemic that occurred in several Latin American countries. Chagas' disease (CD) is an infectious disease caused by the etiological agent Trypanosoma cruzi resulting from human changes in the environment. With the disease ongoing, there is a progressive aggression to the heart, characterized by myocarditis, resulting in chronic Chagas disease cardiomyopathy (CCDC). The acute phase of this disease starts right after the incubation period, which occurs 1-4 weeks after exposure to T. cruzi, the period in which there is no clinical manifestation after contamination is called the incubation period. As it is an infectious disease, the etiological diagnosis of Chagas' disease in Brazil has to be carried out in all suspected cases, in the acute and chronic phases. Thus, it is essential to integrate epidemiological, clinical and laboratory evidence in order to increase the degree of prediction and the accuracy of the diagnosis.

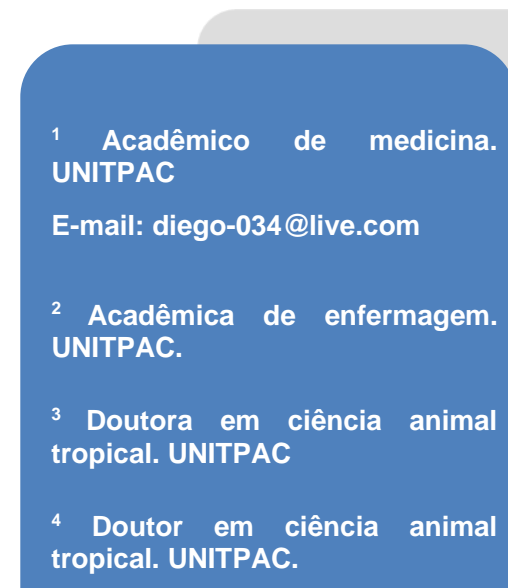

tropical. UNITPAC.

Keywords: Acute Chagas disease. Trypanosoma cruzi. Barber. 


\section{INTRODUÇÄAO}

Os conhecimentos sobre a doença de Chagas tiveram seu pontapé inicial no ano de 1909, através de uma expressiva endemia rural que ocorreu em vários países latinoamericanos (MALAFAIA; DE LIMA RODRIGUES, 2010).

O doutor Carlos Chagas realizou investigações científicas sem os recursos tecnológicos disponíveis atualmente. Foi ele o responsável por evidenciar pela primeira vez a existência de protozoário que ganhou a designação de Trypanosoma cruzi (TATTO; PADILHA; FERNANDES, 2007).

A doença de Chagas (DC) é uma patologia de cunho infeccioso, provocada pelo agente etiológico Trypanosoma cruzi e que provoca entre outras alterações, uma agressão progressiva ao coração. Foi classificada pela Organização Mundial da Saúde (OMS) como uma doença tropical e atualmente, devido as estratégias de controle global encontra-se em declínio e com eliminação programada (TATTO; PADILHA; FERNANDES, 2007).

A doença de Chagas aguda (DCA) é resultante das alterações humanas no meio ambiente, sendo, dessa forma, classificada como uma antropozoonose. Antigamente, o agente etiológico se limitava ao ambiente silvestre, completando seu ciclo biológico em mamíferos provenientes do ambiente silvestre. Através da evolução e avanço do ser humano sobre o meio ambiente, através da destruição da vegetação natural, os triatomíneos modificaram seu comportamento, passando a habitar casas de pau a pique e locais de criação de animais (KROPF; AZEVEDO; FERREIRA, 2000).

Ocorre uma agressão progressiva ao coração, caracterizada por uma miocardite, tem como resultado a cardiomiopatia crônica da doença de Chagas (CCDC). O comprometimento cardíaco da doença, na fase crônica, envolve relevante morbidade e mortalidade, sendo a principal causa de cardiomiopatia não-isquêmica na América Latina (BOZELLI et al., 2006).

Os casos mais graves de DC são caracterizados por comprometimentos cardíacos e digestivos. Ela pode ser transmitida aos indivíduos de variadas formas, entre elas: Vetorial, oral, transfusional, dentre outras (VARGAS et al., 2018).

O período em que não há manifestação clínica após a contaminação é chamado de período de incubação. Ele varia de acordo com a forma de transmissão: Se for transfusional varia de 30 a 40 dias ou mais; Vetorial: 4 a 15 dias; Vertical ela pode ser 
transmitida em qualquer período da gestação ou durante o parto. Oral: 3 a 22 dias. Acidental: até aproximadamente 20 dias (OLIVEIRA et al., 2006).

Inicialmente, na fase aguda da doença, o dano orgânico é estritamente associado à invasão e multiplicação parasitária no miocárdio, e de outros tecidos comumente acometidos como o sistema nervoso e aparelho digestivo. A linfadenopatia e o hepatoesplenomegalia devem-se à reação imunológica sistêmica e correlacionam-se com a elevada parasitemia (SANGENIS et al., 2016).

A fase aguda desta doença inicia-se logo depois do período de incubação, que ocorre de 1-4 semanas após a exposição ao T. cruzi. Ocorrem as lesões conhecidas como "chagomas", o sinal de Romaña. Essas alterações anatômicas decorrem de edema de mucosa ou cutâneo no local da inoculação (PINTO et al., 2007).

$\mathrm{Na}$ fase aguda da doença ocorre um predomínio do parasita na forma circulante, na corrente sanguínea, de maneira expressiva. Ocorre manifestação febril, sendo uma característica peculiar de ser uma febre arrastada. podendo persistir por até 12 semanas (LIMA; TEIXEIRA; LIMA, 2019).

Mesmo que não for tratada, ocorrerá o desaparecimento da febre de forma espontânea e de quase todas as outras manifestações. Já é possível nessa fase realizar a detecção dos anticorpos IgM. Essa detecção é coincidente com a queda da parasitemia circulante, com aumento gradual de anticorpos $\lg G$ ( $4^{\underline{a}}$ à $6^{\underline{a}}$ semana de infecção) (CAVALCANTI et al., 2019).

No Brasil, o consumo de carne de caça era, nos tempos remotos, uma fonte natural de alimento das diversas etnias indígenas e, nos tempos atuais, ainda faz parte da alimentação de tribos que habitam a Região Amazônica (SANGENIS et al., 2016).

A transmissão oral é, nos dias de hoje, uma das principais formas de transmissão da doença no Brasil, particularmente na Região Amazônica (VINHAES; DIAS, 2000).

Os testes sorológicos costumam ser negativos nas primeiras semanas da infecção. O diagnóstico é realizado pela observação dos parasitas circulantes ou seu material genético (PCR), através de hemocultura ou visualização direta do parasita no sangue periférico entre outros inúmeros métodos (ALVES et al., 2018).

Como é uma doença infecciosa, o diagnóstico etiológico da doença de Chagas no Brasil tem que ser realizado em todos os casos suspeitos, na fase aguda e na fase crônica. Desse modo, é fundamental integrar evidências epidemiológicas, clínicas e 
laboratoriais, a fim de se aumentar o grau de predição e a acurácia do diagnóstico (ALVES et al., 2018).

No Brasil, o número reduzido de estudos sistemáticos, de base populacional, dificulta a avaliação por estimativas da magnitude da doença de Chagas ao longo da história (MALAFAIA; DE LIMA RODRIGUES, 2010).

A área com risco de transmissão vetorial para doença de Chagas no Brasil conhecida no final dos anos 1970 incluía 18 estados, com mais de 2.200 municípios, nos quais se comprovou a presença de triatomíneos domiciliados. Ressalta-se que a Região Amazônica estava excluída desta área de risco (ARAS et al., 2003).

A transmissão por via oral é considerada como mecanismo primário, em especial no ciclo silvestre, e seguirá ocorrendo independentemente das ações de controle empreendidas (PINTO et al., 2012).

Atualmente no território brasileiro predomina a forma crônica da doença de Chagas, que são decorrentes de infecção por via vetorial (em torno de 2 milhões). Entretanto, nos últimos 10 anos, a ocorrência de casos e surtos de doença de Chagas aguda (DCA) pode ser observado em vários estados brasileiros, tendo uma maior frequência na Região Amazônica (OLIVEIRA et al., 2006).

Uma recente metanálise trouxe como estimativa de $2,4 \%$ a prevalência da DC no Brasil, o que equivale a 4,6 milhões de indivíduos infectados. Ademais, observou-se o acréscimo do número de transmissões pela via oral através da ingestão de alimentos infectados por triatomíneos infectados por T. cruzi (MALAFAIA; DE LIMA RODRIGUES, 2010).

A Organização Mundial de Saúde estima em 300.000 o número de novos casos por ano na América Latina e acredita que existam 8 milhões de pessoas infectadas em todo 0 mundo (CAVALCANTI et al., 2019).

No Brasil, em relação ao contexto epidemiológico dos eventos de doença de Chagas aguda por transmissão oral, a maior parte dos casos foi relatada na Amazônia em surtos de contextos familiares ou multifamiliares (VINHAES; DIAS, 2000).

Dessa forma, é imperioso que se busque mais conhecimento relativo a cenários epidemiológicos da doença de Chagas e sua forma de transmissão, que envolve pessoas infectadas e sob risco de infecção, diferentes populações do parasita, espécies do vetor e reservatórios de T. cruzi. Este conhecimento adquirido e integrado representa fator central para que se busque ações consistentes e sustentáveis de gestão, vigilância, controle e 
atenção à saúde e social, coerentes, eficazes, efetivas e eficiente (CAVALCANTI et al., 2019).

\section{MATERIAIS E METODOS}

Este foi um estudo epidemiológico retrospectivo com abordagem quantitativa e analítica, que utilizou o sistema informatizado de dados das notificações de doença de Chagas aguda, vinculado à Secretaria Municipal de Saúde de Araguaína-TO e ao DATASUS, abrangendo o período entre 2007 a 2018.

Esse banco de dados é constituído por todos os casos de doença de Chagas aguda notificados e confirmados em Araguaína-TO, através da Ficha Individual de Notificação/Investigação de doença de Chagas aguda, arquivada no Sistema de Informação de Agravos de Notificação (SINAN).

Os critérios de inclusão foram as notificações de casos novos em pacientes com diagnóstico de doença de Chagas aguda, na cidade de Araguaína-TO, registradas no SINAN, no período escolhido. Os critérios de exclusão foram as notificações duvidosas, incompletas, com registro de outro período e as variáveis não selecionadas para este estudo.

As variáveis do estudo foram divididas em oito categorias de análise Casos confirmados por: Mês $1^{\text {a }}$ sintoma, por zona de residência, por UF de infecção, por autóctone, modo provável de infecção, local provável de infecção, faixa etária, escolaridade, raça, sexo, gestante, critério clínico-epidemiológico, por evolução.

A escolha da amostra do estudo é correspondente às pessoas que foram acometidas por doença de Chagas aguda no período escolhido. A busca e coleta dos dados foi realizada de forma gradual, em etapas. Após coletados, os dados foram digitados em planilhas do Excel 2019. Para a análise estatística, os dados foram apresentados em forma de gráficos com frequência absoluta em na discussão foram apresentados em frequência relativa.

O SINAN recebe informações através das fichas de notificação e investigação dos casos de patologias e agravos, que estão na lista nacional de patologias de notificação compulsória, dentre elas a doença de Chagas aguda. Cada caso novo deve ser obrigatoriamente informado na semana epidemiológica de ocorrência pelos profissionais que prestarem atendimento ao paciente. 
Este estudo constitui-se em uma pesquisa documental e eletrônica, portanto, não houve necessidade de aprovação pelo Comitê de Ética em Pesquisa.

\section{RESULTADOS}

De acordo com os dados do Sinan, foram notificados, entre os anos de 2007 e 20018 em Araguaína-TO, 15 casos de doença de Chagas aguda (DCA). Desses casos, pode-se observar que $26,66 \%$ ocorreram no primeiro semestre dos anos analisados e, consequentemente, $73,33 \%$ no segundo semestre. A figura 1 apresenta a distribuição dos casos confirmados por mês de 1aㅗ sintoma segundo município de notificação e período.

Pode-se verificar que a distribuição sazonal dos casos notificados e confirmado de DCA ocorreu de maneira mais significativa nos segundos semestres dos anos analisados, destacando-se os meses de outubro - sendo a região mais seca do ano na região analisada - e que tem como característica altas temperaturas com baixos níveis pluviométricos. Outra característica é a maior ocorrência de queimadas e desflorestamentos o que resulta em aumento de temperatura em determinadas regiões da região.

Dessa maneira, pode-se relacionar o número de casos de DCA com as altas temperaturas nos segundos semestres, estando dessa maneira provavelmente relacionado com à dispersão dos agentes causadores da doença. Dessa maneira, eles se deslocam de seu ambiente silvestre e rumam ao peridomicílio humano o que torna maiores as chances de transmissão vetorial.

Outro ponto que vale destacar é que no segundo semestre do ano é o que ocorre as maiores safras de açaí, que, de uma maneira ou de outra poderá ser contaminado pelo agente causador da DCA e acabar por via oral realizando a contaminação dos pacientes. 


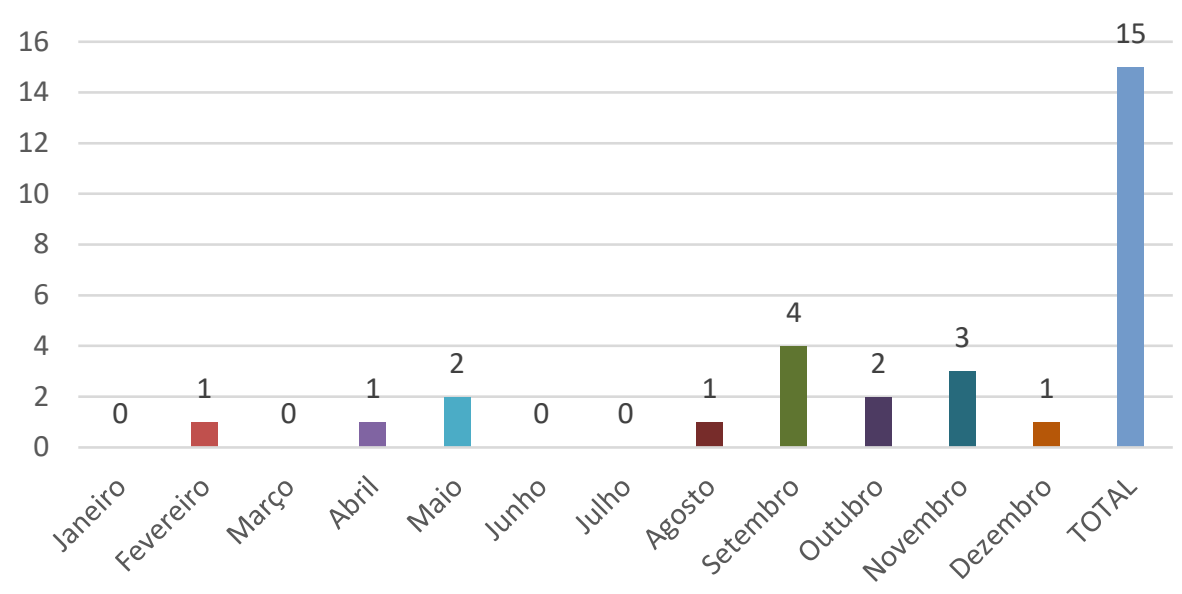

Figura 1: Distribuição dos casos confirmados por mês de $1^{\text {a }}$ sintoma segundo município de notificação e período de 2007 a 2018.

Através deste estudo que a quantidade de casos de DCA quando relacionado ao sexo não mostrou significativa diferença estatística, não podendo ser possível fazer relação risco para contaminação relacionada a essa variável. Ocorreram 8 casos em homens $(53,33 \%)$ e 7 casos em mulheres $(46,67 \%)$ dos casos em mulheres, conforme exposto na figura 2.

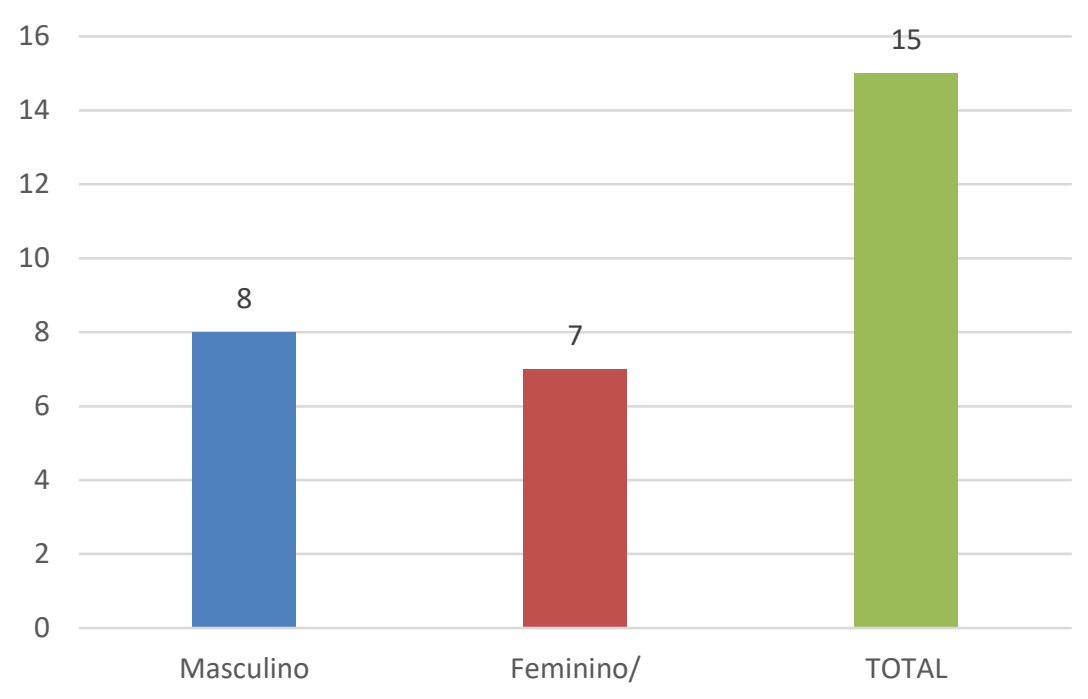

Figura 2: Casos confirmados por sexo segundo município de notificação no período de 2007 a 2018.

Analisando os casos de DCA relacionados com a etnia (figura 3) pode-se observar a prevalência de DCA em pardos com 10 casos registrados relacionados a essa variável $(66,66 \%)$. Pode-se relacionar esse acontecimento devido a sua fragilidade social. 


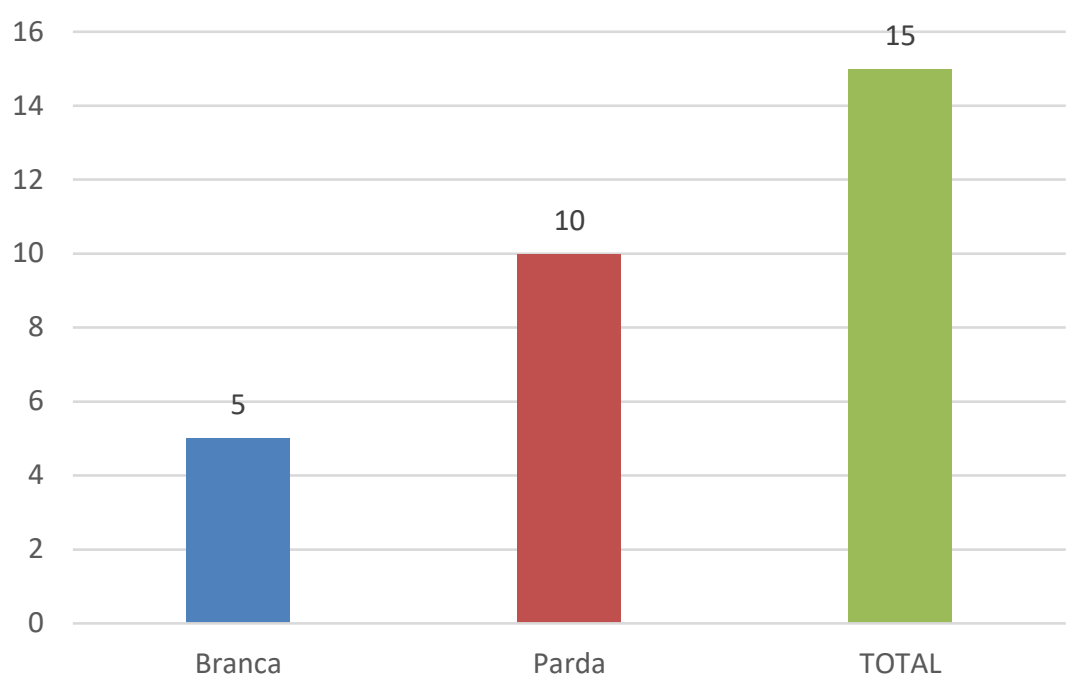

Figura 3: Casos confirmados por raça segundo município de notificação no período de 2007 a 2018.

Quanto à faixa etária, pode-se observar na figura 4, a maioria dos indivíduos acometidos pela DCA está em idade economicamente ativa de 20 a 59 anos, sendo10 casos $(66,66 \%$ dos casos analisados). Isso sugere que possa existir relação entre atividade econômica, atividades laborais e os fatores de risco para transmissão da DCA. Dessa forma, pode-se concluir que a exposição das pessoas que vivem em zonas rurais com o convívio com os vetores contaminados com o patógeno, por meio da extração do açaí, da agricultura familiar, entre outras atividades. Soma-se a isso o fato anteriormente citado de que a sazonalidade da doença ocorre no segundo semestre, período de altas temperaturas, queimadas e desmatamento na região.

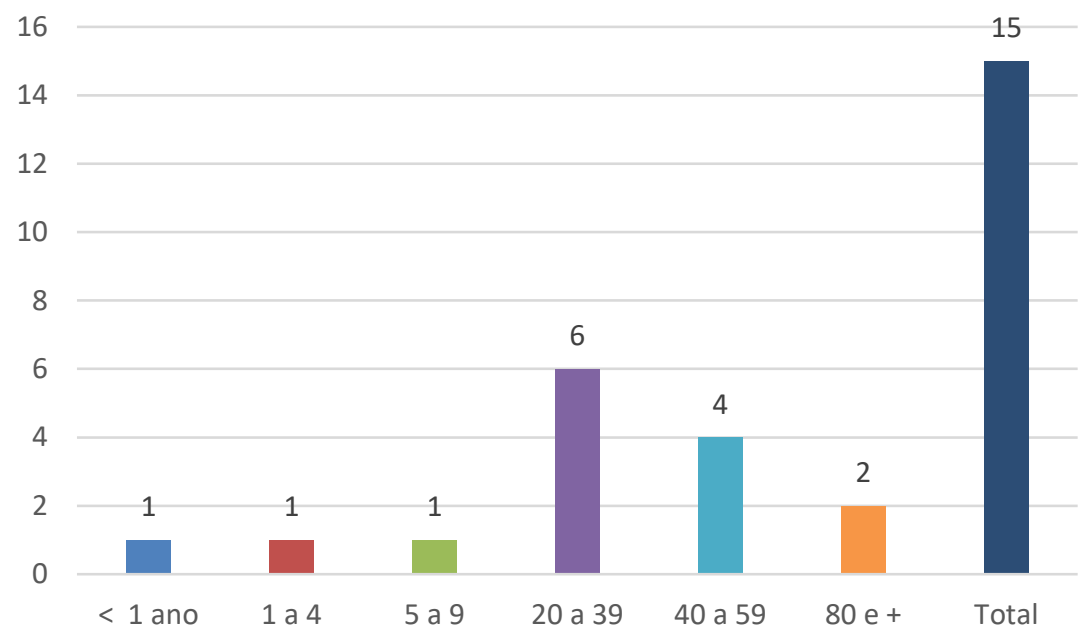

Figura 4: Casos confirmados por faixa etária segundo município de notificação no período de 2007 a 2018. 
No que diz respeito à escolaridade, o maior número de casos (13 casos, sendo $86,66 \%$ do total) não foram especificados no momento da notificação ou foram ignorados (demonstrado na figura 5). Deve-se reforçar a importância para esta variável sendo que, em outros estudos pode-se observar que grade número de casos ocorreu em indivíduos com baixa escolaridade, demonstrando assim a vulnerabilidade social em que estão inseridos esses indivíduos.

Ao mesmo tempo, encontram-se envolvidos em uma situação econômica precária e isso faz com que morem em regiões periféricas, e lá estando, enfrentam a infraestrutura sanitária de péssima qualidade ou inexistente, com baixíssimos níveis de educação básica, sendo assim fator de risco que a expõe aos diversos tipos de transmissão da DC. Dessa forma, vários outros estudos demostraram um expressivo número de pacientes chagásicos apresentou baixa escolaridade, inclusive analfabetismo.

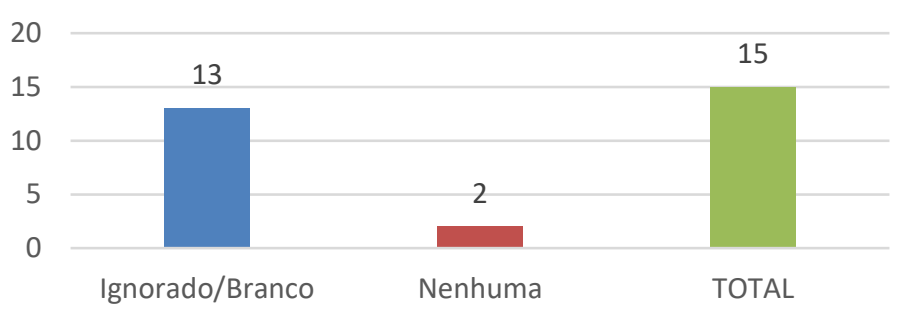

Figura 5: Casos confirmados por escolaridade segundo município de notificação no período de 2007 a 2018.

Outra observação constatada foi a de que uma expressiva parte dos casos confirmados tiveram sua origem na zona urbana do município, correspondendo a 73,33\% do total de casos, indo de encontro com estudos realizados na Amazônia e em outros municípios da região Norte do Brasil. Houve uma mudança no perfil de acometimento da doença, de forma que que foram registrados casos de DCA isolados ou de acometimento familiares (microepidemias), conforme pode ser visualizado na figura 6 .

Atualmente existe no Brasil uma dinâmica de ocupação que implica a formação de novas ocupações urbanas e periurbanas, em localidades que são ambientalmente fragilizadas e com ocorrência da doença nessas localidades, seja por meio oral ou vetorial. 
Observa-se desse modo, uma alteração no perfil epidemiológico da DCA, que em tempos passados era restrita a ambientes silvestres, e atualmente evidencia um processo de urbanização da doença.

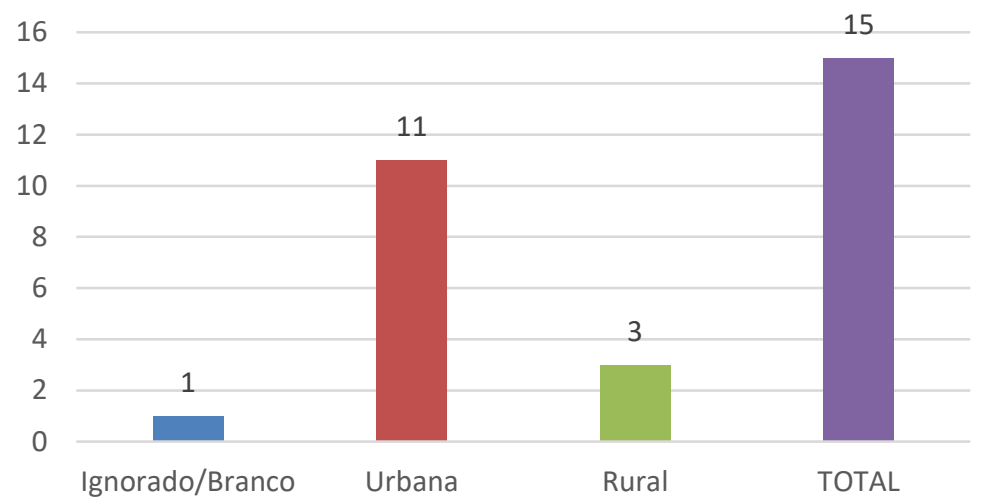

Figura 6: Casos confirmados por Zona de Residência segundo Município de notificação no período de 2007 a 2018.

Outra variável analisada foi relacionada à provável fonte de transmissão (oral ou vetorial). O maior número de casos, sendo 8 no total $(53,33 \%)$, está relacionado a forma oral, que pode estar diretamente relacionada ao consumo de tradicional de suco e polpa de frutas regionais contaminados, principalmente o açaí e bacaba, conforme evidenciado na figura 7.

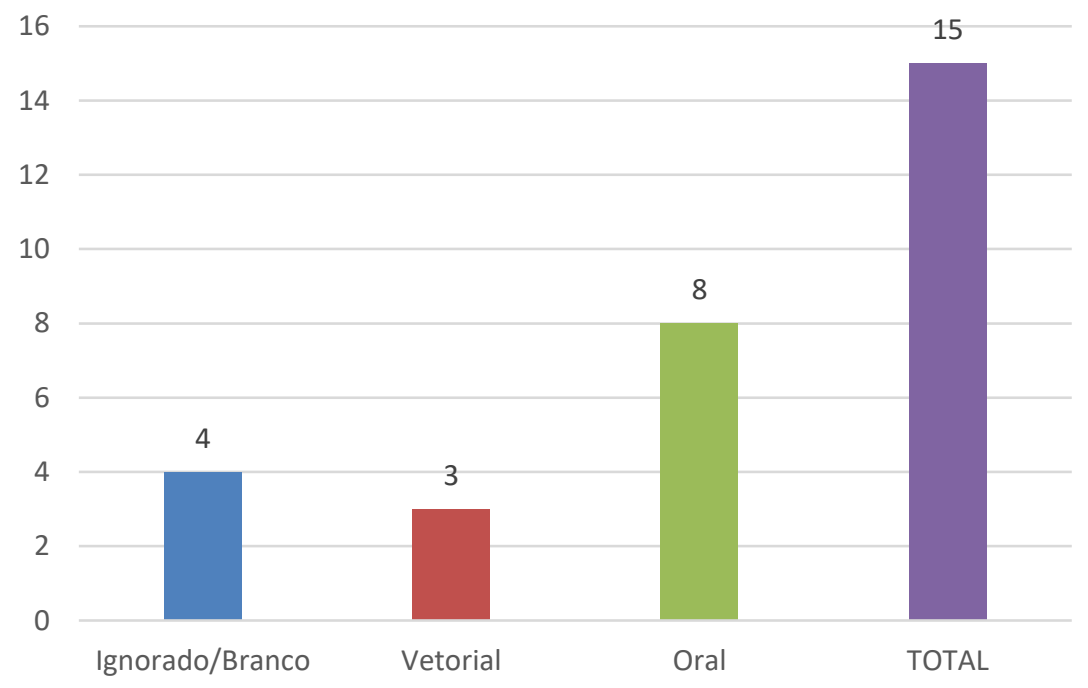

Figura 7: Casos confirmados por modo provável de infecção no período de 2007 a 2018.

Os casos notificados em Araguaína-TO no período analisado tiveram como UF de infecção os estados do Tocantins e Pará, com 9 e 4 casos respectivamente. A cidade de 
Araguaína-TO como um polo industrial e econômico muito importante na região norte do Brasil, tornou-se referência também na questão de procura para resolução de problemas de saúde da população dos estados do Pará e Maranhão.

Por esse motivo, 26,66\% dos casos de DCA notificados no período analisado foram provenientes do estado do Pará. Provavelmente de pessoas que tem parentes, amigos ou outras pessoas que moram em Araguaína e que lhes possam dar assistência na busca por saúde nesse município.

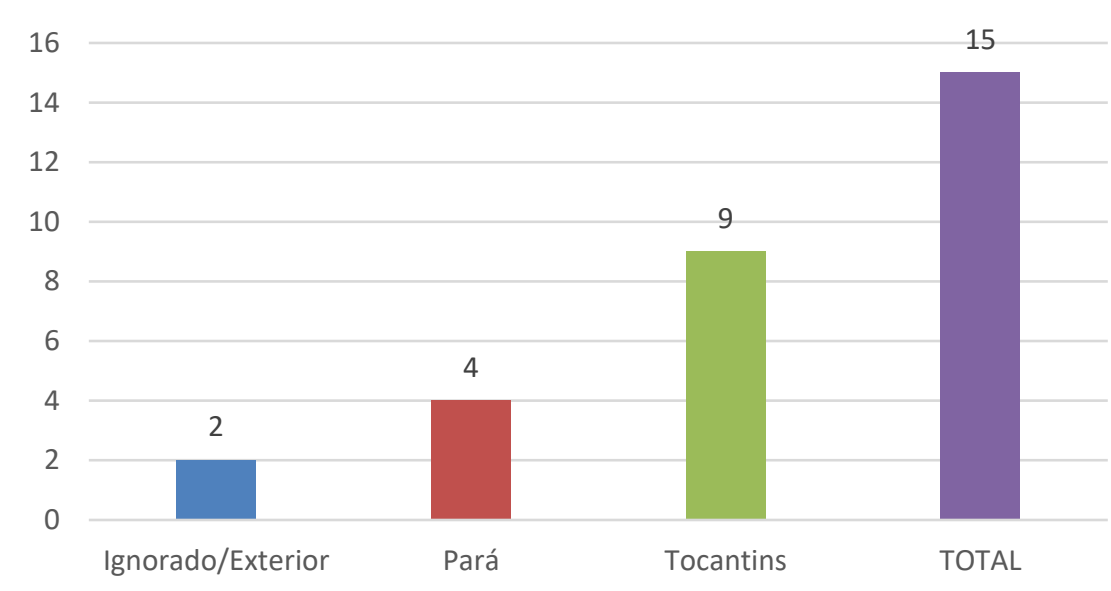

Figura 8: Casos confirmados por UF de infecção segundo município de notificação no período de 2007 a 2018

Do total de casos analisados, 5 deles $(33,33 \%$ do total) foram de pacientes oriundos de Araguaína-TO (autóctones). Com uma área total de extensão de $4.000 \mathrm{~km}^{2}$, possui vários munícios limítrofes e áreas de assentamento rural. Outra característica é que na região é muito comum o consumo de açaí in natura, por meio do qual pode ocorrer a transmissão oral da doença de Chagas. 


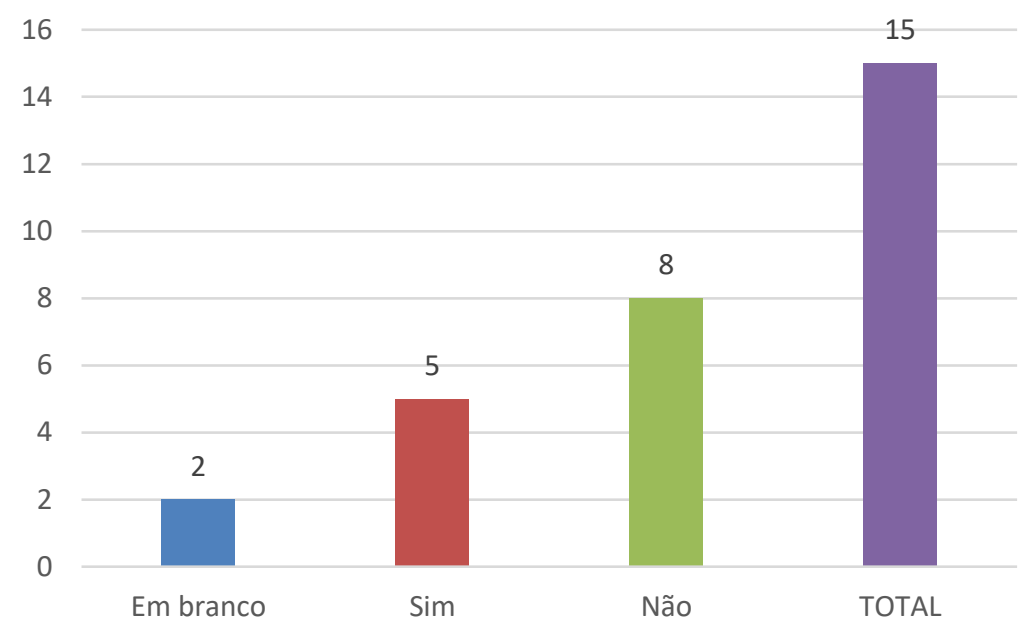

Figura 9: Casos confirmados por autóctone notificados no sistema de informação de agravos de notificação de Araguaína no período de 2007 a 2018.

Outra observação realizada foi a de que em 5 casos (33,33\%) a infecção ocorreu em domicílio, 6 casos não tiveram definidos o local de ocorrência (40\%) e 4 casos ocorreram em locais não especificados (26,66\%) conforme figura 10 . É importante o reconhecimento do local de ocorrência para que se possa realizar uma busca ativa da origem infecciosa dos casos notificados e, dessa maneira, possa evitar que ocorram novas infecções em outros indivíduos da localidade.

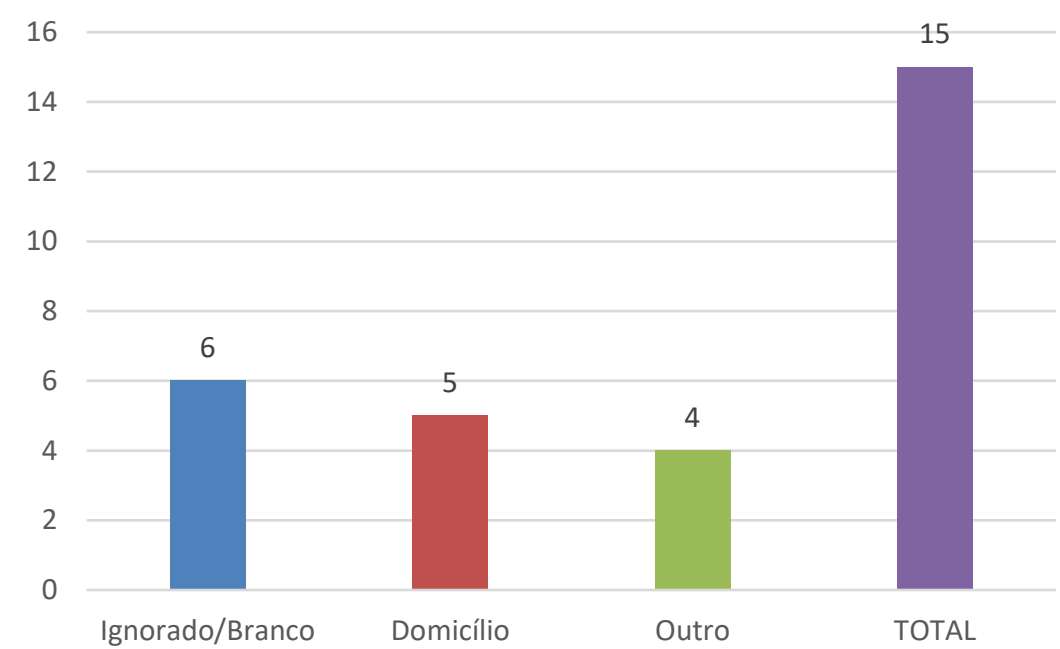

Figura 10: Casos confirmados por local provável de infecção segundo município de notificação no período de 2007 a 2018.

Quando buscadas variáveis relacionadas a gestantes o que se pôde verificar é que houve apenas 1 caso notificado relacionado, e a gestante encontrava-se no segundo 
trimestre de gestação. Os demais casos foram classificados como sendo de 4 em não gestantes e 10 não foram especificados no momento na notificação.

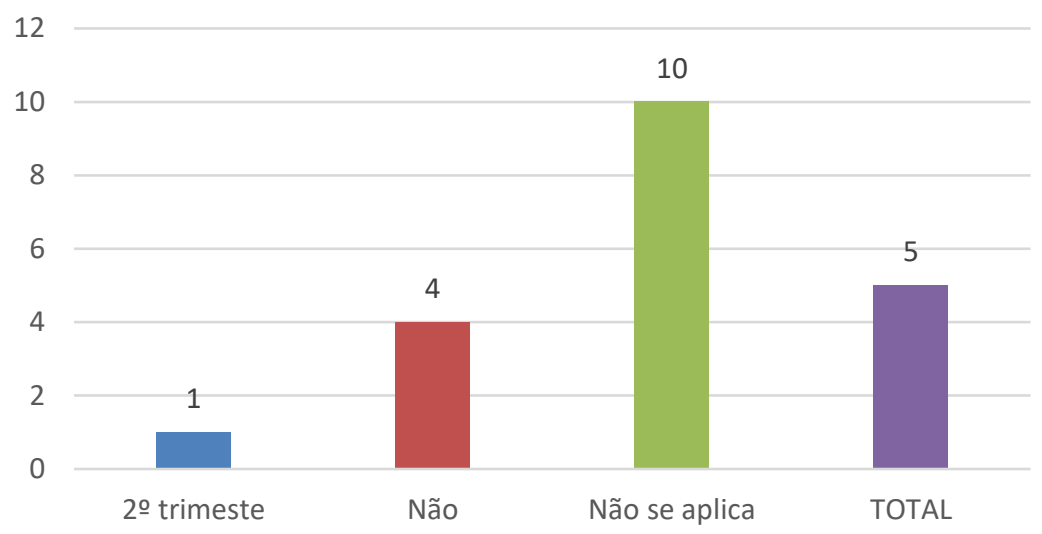

Figura 11: Casos confirmados por gestante segundo município de notificação no período de 2007 a 2018.

Os casos de doença de Chagas aguda tem de ser confirmados através do diagnóstico laboratorial. Entretanto, em condições eventuais, adota-se o critério clínicoepidemiológico para casos suspeitos e que estejam com os exames parasitológicos negativos e sorológicos inicialmente não reagentes e possam ter vínculo epidemiológico com outros casos anteriormente confirmados de DCA por critério laboratorial, durante surto de doença na fase aguda por transmissão oral.

Dessa forma, no período analisado pode-se observar que 93,33\% (14 casos) de todos os casos foram confirmados por meio laboratorial e apenas $6,66 \%$ deles ( 1 caso) foi realizado o diagnóstico pelo meio clínico-epidemiológico, corroborando com a literatura atual que coloca o diagnóstico laboratorial como principal meio para elucidação dos casos. 


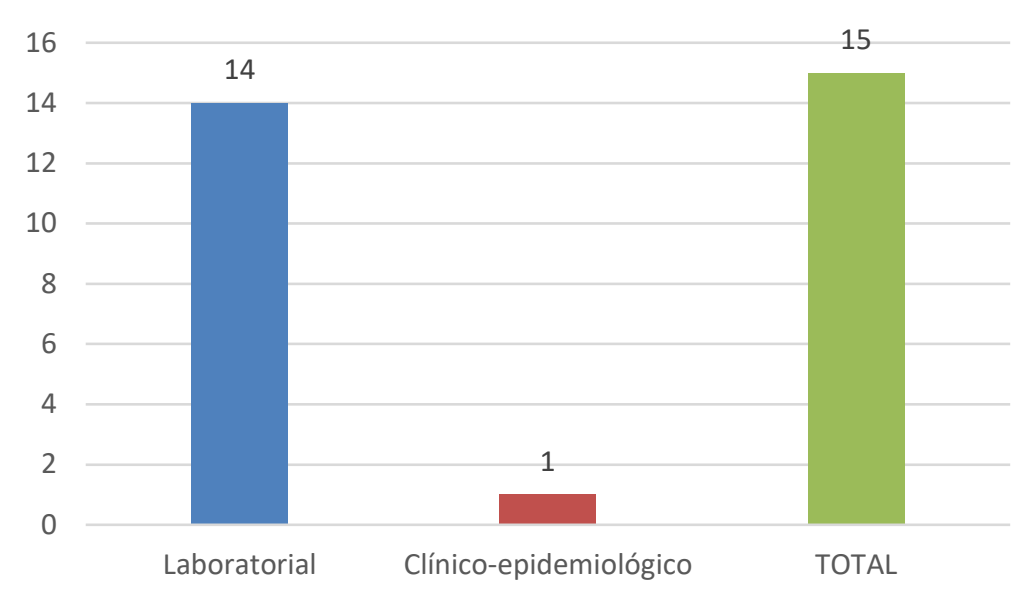

Figura 12: Casos confirmados por critério clínico-epidemiológico ou laboratorial no período de 2007 a 2018.

A última variável analisada foi a evolução dos casos no período estudado. Desses, $93,33 \%$ evoluíram com tratamento e seguimento da doença e apenas 6,66\% (1 caso) teve evolução fatal como desfecho da DCA.

Durante o desenvolvimento dessa pesquisa observou-se algumas limitações no que diz respeito a análise das variáveis escolhidas. Esse fato tem íntima relação com o processo inadequado de notificação dos dados no sistema de vigilância epidemiológica do município.

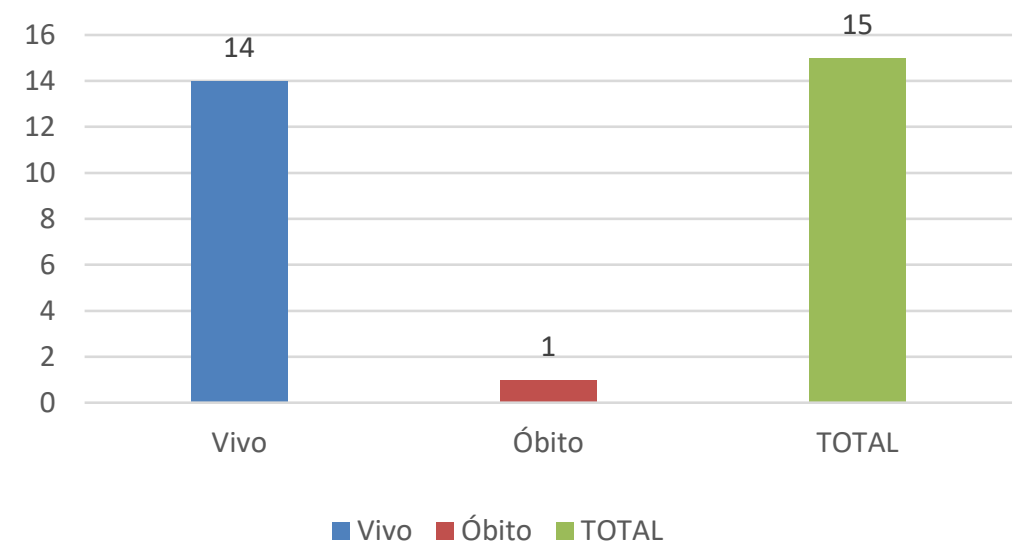

Figura 13: Casos confirmados por evolução no período de 2007 a 2018.

\section{CONSIDERAÇÕES FINAIS}

Durante a realização desta pesquisa observou-se que existem diversas relações epidemiológicas associadas a notificação de DCA no município de Araguaína-TO, como 
por exemplo, variáveis epidemiológicas, ambientais e demográficas, no período de 2007 a 2018.

Dentre as constatações realizadas, observou-se sazonalidade com maior ocorrência de casos nos segundos semestres, concomitante as altas temperaturas e baixos índices pluviométricos na região. O perfil socioepidemiológico dos indivíduos acometidos pela doença foi: Pessoas de baixa escolaridade, economicamente ativas (adultas), pardas, com possível forma de transmissão principalmente por via oral e ter residência, morar em área urbana.

Considerando as diferentes relações das variáveis estudadas, as ferramentas computacionais utilizadas na análise espacial dos dados foram satisfatórias para a construção da ANÁLISE DO PERFIL EPIDEMIOLÓGICO DOS PACIENTE ACOMETIDOS POR DOENÇA DE CHAGAS AGUDA EM ARAGUAÍNA-TO NO PERÍODO DE 2007 A 2018 e verificação dos cenários epidemiológicos em que ocorrem a DCA.

Dessa forma, esse tipo de análise é de suma importância pois apresentam grande potencial para prover os gestores em saúde com informações voltadas para a vigilância contínua e sistemática do agravo estudado.

\section{REFERENCIAS}

ALVES, D. F. et al. Diagnostic methods of Chagas disease: an update. Revista Brasileira de Análises Clínicas, v. 50, n. 4, 2018.

ARAS, R. et al. Transmissão vetorial da doença de Chagas em Mulungu do Morro, Nordeste do Brasil. Revista da Sociedade Brasileira de Medicina Tropical, v. 36, n. 3, p. 359-363, maio 2003.

BOZELLI, C. E. et al. Perfil clínico-epidemiológico de pacientes com doença de Chagas no Hospital Universitário de Maringá, Paraná, Brasil. Cadernos de Saude Publica, v. 22, n. 5, p. 1027-1034, 2006.

CAVALCANTI, M. A. F. et al. Manifestations and strategies of coping with chagas disease that interfere in the quality of life of the individual: A systematic review. Ciencia e Saude Coletiva, v. 24, n. 4, p. 1405-1416, 1 abr. 2019.

KROPF, S. P.; AZEVEDO, N.; FERREIRA, L. O. Moléstia de Carlos Chagas: Conferência realizada em 7 de agosto na Academia Nacional de Medicina. Brazil-Medico,. BrazilMedico, v. 25, p. 347-365, 2000.

LIMA, R. DE S.; TEIXEIRA, A. B.; LIMA, V. L. DA S. Doença de chagas: uma atualização bibliográfica. Revista Brasileira de Análises Clínicas, v. 51, n. 2, 2019. 
MALAFAIA, G.; DE LIMA RODRIGUES, A. S. Centenário do descobrimento da doença de chagas: Desafios e perspectives. Revista da Sociedade Brasileira de Medicina Tropical, v. 43, n. 5, p. 483-485, 2010.

OLIVEIRA, F. A. S. et al. Características epidemiológicas dos pacientes com Doença de Chagas. Revista Brasileira de Medicina de Família e Comunidade, v. 2, n. 6, p. 107113, 2006.

PINTO, A. Y. D. N. et al. Fase aguda da doença de Chagas na Amazônia brasileira. Estudo de 233 casos do Pará, Amapá e Maranhão observados entre 1988 e 2005. Revista da Sociedade Brasileira de Medicina Tropical, v. 41, n. 6, p. 602-614, nov. 2008.

PINTO, A. Y. DAS N. et al. Doença de chagas aguda grave autócne da Amazônia brasileira. Revista Paraense de Medicina, v. 21, n. 2, 2007.

PINTO, V. D. S. et al. Vigilância Epidemiológica da Paracoccidioidomicose no Estado de São Paulo, 2008 a 2011. BEPA - Boletim epidemiológico Paulista, v. 9, n. 103, p. 4-15, 2012.

SANGENIS, L. H. C. et al. Transmissão da doença de Chagas por consumo de carne de caça: revisão sistemática. Revista Brasileira de Epidemiologia, v. 19, n. 4, p. 803-811, 1 dez. 2016.

TATTO, E.; PADILHA, E. MAURA; FERNANDES, S. B. DOENÇA DE CHAGAS AGUDA Aspectos epidemiológicos, diagnóstico e tratamento Guia de consulta rápida para profissionais de saúde. Programa nacional de Controle de Chagas, Secretaria de Vigilância em Saúde, Ministério da Saúde., p. 32, 2007.

VARGAS, A. et al. Investigação de surto de doença de Chagas aguda na região extraamazônica, Rio Grande do Norte, Brasil, 2016. Cadernos de Saúde Pública, v. 34, n. 1, p. 1-8, 2018.

VINHAES, M. C.; DIAS, J. C. P. Doença de Chagas no Brasil. Cadernos de Saúde Pública, v. 16, n. suppl 2, p. S7-S12, 2000. 